Die Geschichte des Zürcher Medizinstudiums steht im Mittelpunkt des Sammelbands «Innenansichten einer Ärzteschmiede», der anlässlich des Universitätsjubiläums im Chronos-Verlag erschienen ist (www.175jahre.uzh.ch/gruenschnabel-weisskittel). Die Schweizerische Ärztezeitung druckt Auszüge aus den Artikeln in einer Serie ab. Der Beitrag, aus dem die heutigen Auszüge stammen, verfolgt die Geschichte der Visualisierungstechniken in der dermatologischen Lehre von den ersten Atlanten bis zur heutigen Computerprojektion.

\title{
Visualisierung in der universitären Lehre am Beispiel der Dermatologie*
}

Michael L. Geiges

* Auszüge aus dem Beitrag des Autors in: Ritzmann I, Schweer W, Wolff E (Hrsg.). Innenansichten einer Ärzteschmiede. Lehren, lernen und leben - aus der Geschichte des Zürcher Medizinstudiums. Zürich: Chronos; 2008. 240 S., 80 teils farbige Abbildungen. Fr. 34.-/€ 20.60. ISBN 978-3-0340-0909-6. Quellenverweise im Originalartikel.

Korrespondenz:

Dr. med. Michael L. Geiges Facharzt für Dermatologie und Venerologie FMH

Universität Zürich

Medizinhistorisches Institut

Hirschengraben 82

CH-8001 Zürich

Tel. 0446342019

michael.geiges@mhiz.uzh.ch

Am 23. Juli 1924 wurden die neuen Räumlichkeiten der Dermatologischen Klinik in Zürich feierlich eröffnet. Mit zum Neubau gehörte ein Auditorium, das Platz für 118 Studierende bot. Der steile Hörsaal erstreckte sich über zwei Stockwerke und war mit technischen Geräten zur Demonstration von Bildern, Moulagen, mikroskopischen Präparaten und Patienten eingerichtet.

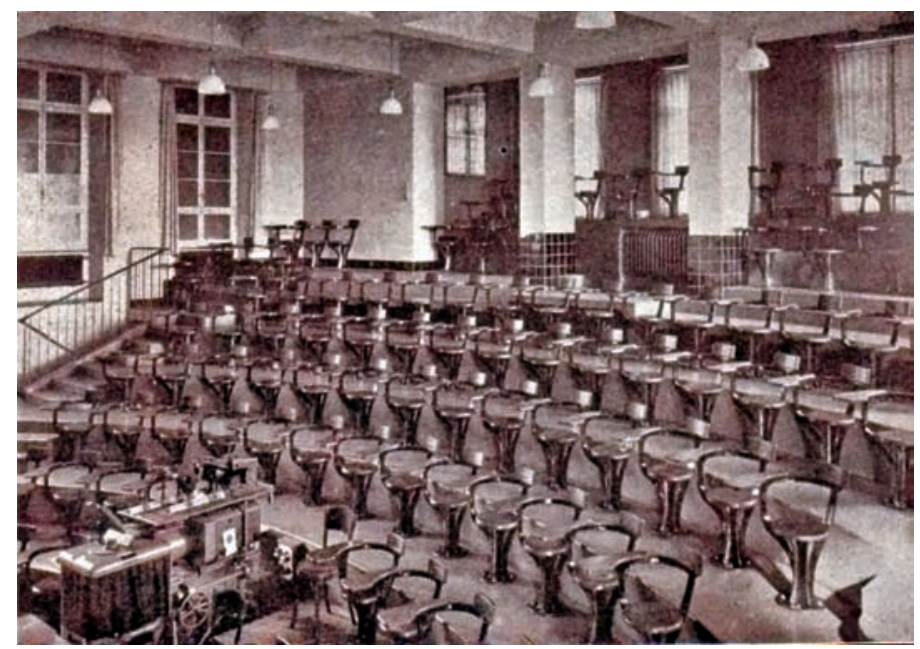

Hörsaal in der Dermatologischen Klinik um 1929 (aus: Bloch B. Die Dermatologische Universitätsklinik Zürich. In: The Rockefeller Foundation (ed.): Methods and Problems of Medical Education. New York; 1929).

Die Ausbildung im Hörsaal stellte ganz neue Anforderungen an das stark visuell orientierte Fach. Für Bloch war klar, dass der Unterricht nur Sinn ergibt, «wenn dem Studenten Gelegenheit geboten wird, möglichst viele Fälle und diese möglichst gründlich und aus der Nähe zu studieren. Die Diagnostik hängt [...] von der Ausbildung des visuellen Sehvermögens ab.»

Die Systematik nach botanischem Vorbild beruhte auf dem Sammeln und Vergleichen von Effloreszenzen (Hautveränderungen). Es war daher wichtig, möglichst viele Patienten und Krankheitsbilder für einen Vergleich nebeneinander sehen zu können, was nur dank der Konzentration von Patienten in Spitälern überhaupt erst verwirklicht werden konnte. Aus vielen Krankheitsfällen mit jeweils individuellen Befunden und Verläufen (Realtypen) konnten so Idealtypen konstruiert werden, die das Charakteristische und Typische einer Hautkrankheit betonen.

Die Demonstration von Pa-

Bruno Bloch (1878-1933), der erfolgreiche Direktor der erst jungen Klinik, legte grosses Gewicht auf die direkte Patientendemonstration. Dies spiegelte sich in der Architektur dieses Hörsaals, der seinen Vorgaben gemäss gebaut worden war. Damit die Studierenden nicht mit den Patienten in Berührung kamen, gelangten sie durch einen eigenen Eingang ins Gebäude. Zudem wurde der Hörsaal so eingerichtet, «dass jeder Kranke, ohne Störung des Vortrages, von den Assistenten durch die Zwischenräume der Bankreihen hindurch geführt und jedem Studenten einzeln vordemonstriert werden kann». tienten in der Vorlesung und in Kursen hatte Nachteile und Grenzen, wie Alfred Stühmer (1885-1957), Dermatologieprofessor in Freiburg/Br., 1958 formulierte: «Die grosse Zahl der Hörer macht es unmöglich, die Einzelheiten des besonderen Falles jedem Studenten vor Augen zu führen.» Insbesondere bei einer «Hörerzahl von 100 und mehr» müsse die unmittelbare Demonstration am Kranken fallengelassen werden.

Obschon Stühmer explizit die Meinung vertrat, die unmittelbare Besichtigung der Kranken könne nicht durch bildliches Unterrichtsmaterial ersetzt werden, traten Bilder in der Lehre mehr 
und mehr an die Stelle von Patienten. Vorerst griff man nur auf Bilder zurück, wenn Beispiele für die besprochenen Krankheiten im Spital fehlten oder wenn Patienten nicht allen Studierenden aus der Nähe gezeigt werden konnten. Schliesslich fand aber auch die Situation der «ausgestellten» Patienten mehr und mehr Beachtung. An Kongressen wurde ab 1970 die «Live-Demonstration» endgültig durch die «Dia-Klinik» ersetzt. Nicht so im kleineren Rahmen der medizinischen Vorlesung: Dort begleitet die direkte Demonstration von Patienten den universitären Dermato- logieunterricht bis auf den heutigen Tag. Die Patientendemonstration im Hörsaal nimmt in Zürich weiterhin einen wichtigen Platz in der Vorlesung ein. Dabei wird der Patient wieder direkt im Hörsaal vom Dozenten begrüsst und befragt, während gleichzeitig der Befund über eine leistungsstarke Videokamera per Beamer an die Wand projiziert wird.

In der nächsten Folge: Studieren per Mausklick - E-Learning an der Medizinischen Fakultät Zürich.

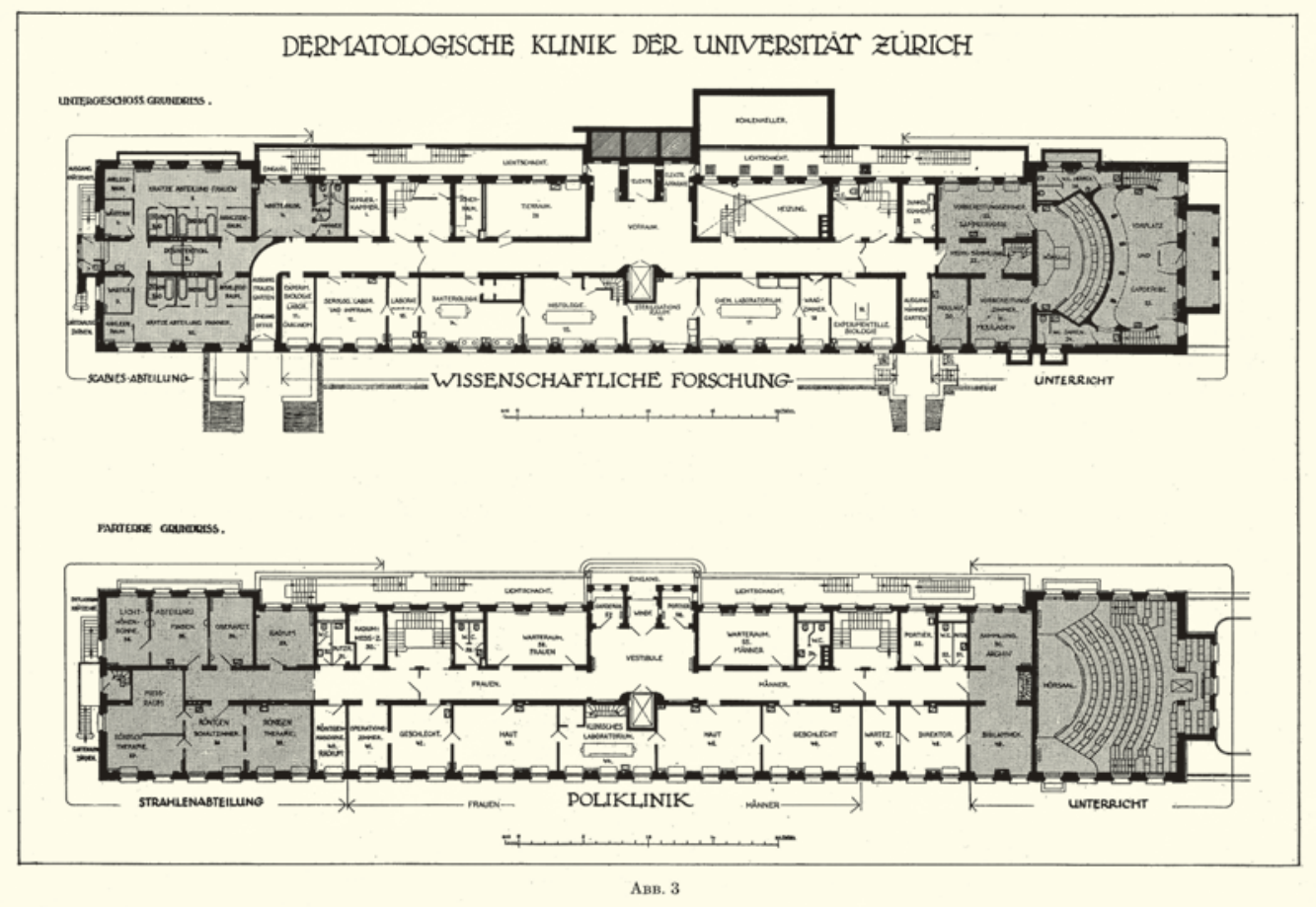

Grundriss der 1924 eröffneten Dermatologischen Universitätsklinik Zürich, rechts Auditorium und Vorbereitungsräume (aus: Bloch B. Die Dermatologische Universitätsklinik Zürich. In: The Rockefeller Foundation (ed.): Methods and Problems of Medical Education. New York; 1929). 